IUHET-485

hep-th/0507258

\title{
Lorentz Violation and Synchrotron Radiation
}

\author{
B. Altschul ${ }^{1}$ \\ Department of Physics \\ Indiana University \\ Bloomington, IN 47405 USA
}

\begin{abstract}
We consider the radiation emitted by an ultrarelativistic charged particle moving in a magnetic field, in the presence of an additional Lorentz-violating interaction. In contrast with prior work, we treat a form of Lorentz violation that is represented by a renormalizable operator. Neglecting the radiative reaction force, the particle's trajectory can be determined exactly. The resulting orbit is generally noncircular and does not lie in the plane perpendicular to the magnetic field. We do not consider any Lorentz violation in the electromagnetic sector, so the radiation from the accelerated charge can be determined by standard means, and the radiation spectrum will exhibit a Lorentz-violating directional dependence. Using data on emission from the Crab nebula, we can set a bound on a particular combination of Lorentz-violating coefficients at the $6 \times 10^{-20}$ level.
\end{abstract}

\footnotetext{
${ }^{1}$ baltschu@indiana.edu
} 


\section{Introduction}

There is presently a great deal of interest in the possibility that Lorentz and CPT invariance may be violated in nature. If the fundamental laws of physics do not obey these symmetries, then we would expect to see evidence of this violation even in the low-energy effective theory. Therefore, if small Lorentz or CPT violations were discovered, they would represent crucial clues about the structure of the most basic theory of nature. A general standard model extension (SME), containing possible Lorentz- and CPT-violating corrections to quantum field theory [1, 2] and general relativity [3] has been developed. The SME offers a parameterization of Lorentz violations in low-energy effective field theory, and both its renormalizability [4] and stability [5] have been carefully examined.

The SME provides a theoretical framework for analyzing experimental results. Sensitive tests of Lorentz symmetry have included studies of matter-antimatter asymmetries for trapped charged particles [6, 7, 8, 9] and bound state systems [10, 11], determinations of muon properties [12, 13, analyses of the behavior of spin-polarized matter [14, 15, frequency standard comparisons [16, 17, 18, measurements of neutral meson oscillations [19, 20, 21, 22, polarization measurements on the light from distant galaxies [23, 24, 25], and others. The results of these experiments can be used to set bounds on various SME coefficients. Many coefficients are very strongly constrained, but many others are not.

There are many systems and reaction processes that could potentially be used to set further bounds of the SME coefficients for Lorentz violation. We shall consider a particular process - synchrotron motion and radiation - and examine how it would be impacted by Lorentz violation. Although there have been many analyses of this process in the presence of Lorentz-violating dispersion relations, there is as yet no analysis in terms of the renormalizable operators of the SME.

Analyses of possible Lorentz violation in synchrotron emission have often focused only on changes to particle dispersion relations. One popular approach is that of Myers and Pospelov [26]. Taking a preferred direction $v^{\mu}$ in spacetime, one may add an operator proportional to $i \phi^{*}\left(v^{\mu} \partial_{\mu}\right)^{3} \phi$ to the Lagrange density for a scalar particle. If $v^{\mu}$ has a time component only, this will add a term proportional to $E^{3}$ to the usual relativistic energymomentum relation $E^{2}=\vec{p}^{2}+m^{2}$. Of course, the statement that $v^{\mu}$ is purely timelike is not Lorentz invariant, so that assumption must be taken to hold is some particular preferred frame, which is typically the rest frame of the cosmic microwave background. The electromagnetic field is incorporated through the usual minimal coupling procedure. In the presence of this kind of Lorentz violation, the motion of a charged particle in a constant magnetic field is modified, but the projection of the trajectory onto the plane

perpendicular to $\vec{B}$ remains circular, and the particle's speed remains constant. The radiation in the far field can be determined, including information about polarization, and circumstances that could enhance observable effects have been identified [27, 28.

Stringent bounds on Lorentz violations with modified dispersion relations have been 
obtained from data from the Crab nebula [29, 30, 31]. These modifications can lead to maximum particle velocities that are less than the speed of light, but the Crab nebula shows evidence of synchrotron emission from electrons with Lorentz factors of $\gamma=$ $\left(1-\vec{v}^{2}\right)^{-1 / 2} \sim 3 \times 10^{9}$, or energies of $1500 \mathrm{TeV}$. So the existence of electrons with velocities this large can be used to constrain models with deformed dispersion relations. For a Lorentz-violating coefficient with a particular sign, the data show that the coefficient must be at least seven orders of magnitude smaller than $\mathcal{O}\left(E / M_{P}\right)$ Planck-level suppression.

Lorentz violation can also be incorporated into particle physics through the introduction of noncommutative field theory [32. Synchrotron radiation has also been analyzed within this framework. The minimal coupling between charged matter and the electromagnetic field is modified by the noncommutativity, as is the structure of the free radiation field itself. A discussion in 33 focuses on the particular case in which the magnetic field and the Lorentz-violating noncommutativity parameter are aligned, so that the orbits of

charged particles in the plane perpendicular to $\vec{B}$ are again given by circles. It is possible to work out the far fields within this model, at leading order in the noncommutativity, but there are a number a difficulties, including acausality and potential problems with quantization.

However, these analyses ignore some of the most natural Lorentz-violating operators. There is a unique spin-independent, superficially renormalizable SME coupling that is consistent with the gauge invariance of the standard model and which grows in relative importance at high energies. This is a CPT-even two-index tensor $c^{\nu \mu}$. We shall look at how the presence of such a constant background tensor (which could arise, for example, as the vacuum expectation value of a dynamical tensor field) will modify synchrotron emission. Using existing data about the nonthermal spectrum of the Crab nebula, we may place a bound of $6 \times 10^{-20}$ on a particular linear combination of the $c^{\nu \mu}$ coefficients. The method by which we find this bound is very similar to that used to bound other types of Lorentz violation; however, the $c^{\nu \mu}$ interaction is actually much more natural to consider than these, because it is superficially renormalizable. All the analyses so far have been essentially classical in nature, and we shall continue working within the classical framework, although we shall look at when quantum corrections would become important.

\section{Synchrotron Motion with Lorentz Violation}

To study synchrotron motion, we shall consider a theory of fermions interacting with the electromagnetic field. The Lagrange density for this theory is

$$
\begin{aligned}
\mathcal{L} & =-\frac{1}{4} F^{\mu \nu} F_{\mu \nu}+\bar{\psi}\left[\Gamma^{\mu}\left(i \partial_{\mu}-e A_{\mu}\right)-m\right] \psi \\
& =-\frac{1}{4} F^{\mu \nu} F_{\mu \nu}+\bar{\psi}\left[\left(\gamma^{\mu}+c^{\nu \mu} \gamma_{\nu}\right)\left(i \partial_{\mu}-e A_{\mu}\right)-m\right] \psi
\end{aligned}
$$


The $c^{\nu \mu}$ interaction is the source of the Lorentz violation. There are other superficially renormalizable couplings contained in the Standard Model Extension, but the $c$ couplings are most natural in this context. When considering synchrotron radiation, one is primarily interested in particles with very high energies. Lorentz-violating coefficients that modify the kinetic part of the Lagrangian will grow in relative importance at high energies, so it is natural to consider only these kinetic modifications. There are only two such sets of Lorentz-violating terms that are consistent with the more general standard model gauge couplings - the $c$ terms and also a set of $d^{\nu \mu}$ terms, which have the same form as the $c$ interactions, except for the addition of a $\gamma_{5}$.

However, we shall not consider the $d$ interactions here. They are spin-dependent, while the $c$ term exists for bosonic (Klein-Gordon) particles as well as fermions. So all our results will apply equally to the motion of spin-zero charged particles. Moreover, spin precession effects will naturally decrease the importance of any $d$ terms. For an electron undergoing circular cyclotron motion, with the spin oriented in the plane of the orbit, the spin rotates by $2 \pi \gamma \frac{g-2}{2}$ radians with each orbital revolution. For $\gamma \gg \alpha^{-1}$, the spin will rotate many times during one orbital period, and any effects proportional to the helicity will be diminished by the resultant averaging.

Modifications of the kinetic Lagrangian that are not invariant under the standard model's $S U(2)_{L}$ gauge symmetry can also exist; however, they can only appear as part of electroweak symmetry breaking, as vacuum expectation values of nonrenormalizable operators. These operators should therefore be further suppressed, and we shall neglect them.

We shall also neglect any Lorentz violation in the photon sector. Modifications of the free electromagnetic Lagrangian will generally change the speed of photon propagation. This leads to the possibility of vacuum Cerenkov radiation [34, 35, which is not yet fully understood, although threshold analyses can be used to set further limits on Lorentzviolating parameters. Most possible Lorentz-violating terms in the free electromagnetic sector also give rise to photon birefringence, which has been searched for and not seen. The limits on the relevant forms of Lorentz violation are very strong, and we may safely neglect them. The purely electromagnetic terms that do not cause birefringence can be accounted for by adding

$$
\mathcal{L}_{F}=-\frac{1}{4}\left(k_{F}\right)_{\mu \alpha \nu}^{\alpha}\left(F^{\rho \mu} F_{\rho}{ }^{\nu}+F^{\mu \rho} F_{\rho}^{\nu}\right) .
$$

to $\mathcal{L}$. However, a coordinate transformation $x^{\mu} \rightarrow x^{\mu}-\frac{1}{2}\left(k_{F}\right)^{\alpha \mu}{ }_{\alpha \nu} x^{\nu}$ will eliminate all the Lorentz violation from the photon sector at leading order [36, 37]. This transformation shifts the Lorentz-violating physics into the charged matter sector, where it manifests itself exactly as a $c^{\nu \mu}$ term. We see that consideration of $c$ therefore captures all the possible sources of Lorentz violation in a synchrotron process that are not significantly further suppressed. However, the transformation that eliminates $k_{F}$ is frame-dependent, and the new coordinates need not even be rectangular relative to the original ones; so by 
choosing to consider only this form of Lorentz violation, we are restricting ourselves to working in a very particular and special coordinate system.

We know that the Lorentz-violating coefficients for any physical charged particles are small. Physically, we might expect that the characteristic size for $c^{\nu \mu}$ is $\mathcal{O}\left(m / M_{P}\right)$. However, we shall not make any special use of the fact that $M_{P}$ is the Planck scale. Rather, we may take this size estimate as effectively defining $M_{P} ; M_{P}$ is whatever large energy scale is needed in order to give the $c$ terms the correct magnitude.

The canonical quantization of the fermion field requires some care when the $c$ coefficients are nonvanishing. If $c^{\nu 0}$ is nonzero, then $\mathcal{L}$ will contain nonstandard time derivative terms. In this case, a matrix transformation $\psi \rightarrow R \psi$ will be required, to ensure that $\Gamma^{0}=\gamma^{0}$. An explicit power-series expression for the required $R$ is given in [38. For simplicity, we shall assume that any such necessary transformation has already been performed and $c^{\nu 0}=0$. However, this will require us to consider the canonical quantization in a single frame only. We may not boost the theory into another frame, because doing so would reintroduce the problematic time derivatives.

In fact, in much of what follows, we shall neglect the $c^{0 \mu}$ terms as well. While there is no special reason to believe this, we shall assume that the Lorentz violation is purely spacelike in a frame in which $F^{\mu \nu}$ contains only a magnetic component. We do this because the problem can then be solved exactly, to all orders in the remaining Lorentz-violating coefficients. However, when we revert to the linearized approximation and derive a limit on the $c$ coefficients from the observed properties of the synchrotron spectrum, we shall include the $c^{0 \mu}$ parts in the calculation.

We shall consider the interaction with the electromagnetic field in two stages. This is standard practice in consideration of cyclotron motion. First, we determine the path traced out by a nonradiating charged particle moving in a spatially homogeneous background magnetic field. Then we evaluate the radiation induced by this periodic motion.

Because we are interested in the synchrotron emission from a single particle, we shall make the natural approximation of treating the Dirac equation as a single-particle wave equation. Standard techniques of relativistic quantum mechanics then apply; however, Lorentz violation will introduce new complexities. The momentum and velocity are not simply related by $\vec{v}=\vec{\pi} / E=\vec{\pi} / \gamma m$. The single-particle fermion Hamiltonian derived from $\mathcal{L}$ is

$$
H=\alpha_{j} \pi_{j}-c_{l j} \alpha_{l} \pi_{j}-c_{0 j} \pi_{j}+\beta m,
$$

where $\alpha_{j}=\gamma^{0} \gamma^{j}$ and $\beta=\gamma^{0}$ are the usual Dirac matrices, and $\vec{\pi}$ is the mechanical (rather than canonical) three-momentum $\vec{\pi}=\vec{p}-e \vec{A}$. Time derivatives of operators relating to fermion properties may be found by taking commutators with this Hamiltonian. In particular, the derivative of the particle's position is

$$
\dot{x}_{k}=\alpha_{k}-c_{l k} \alpha_{l}-c_{0 k} .
$$

Similarly, the equation of motion for $\alpha_{k}$ may be written

$$
\dot{\alpha}_{k}=i\left[-2 \alpha_{k}\left(H+c_{0 j} \pi_{j}\right)+2 \pi_{k}-2 c_{k j} \pi_{j}\right],
$$


which has the exact solution

$$
\alpha_{k}(t)=\left(\pi_{k}-c_{k j} \pi_{j}\right)\left(H+c_{0 j} \pi_{j}\right)^{-1}+\left[\alpha_{k}(0)-\left(\pi_{k}-c_{k j} \pi_{j}\right)\left(H+c_{0 j} \pi_{j}\right)^{-1}\right] e^{-2 i\left(H+c_{0 j} \pi_{j}\right) t} .
$$

The second term on the right-hand-side of (6) is matrix-valued and oscillatory. This term describes the particle's Zitterbewegung, which, for a well-localized wave packet, is generated by interference between positive and negative frequency (i.e. particle and antiparticle) modes. The first term, when combined with (4) gives the bulk velocity [39]

$$
v_{k}=\frac{1}{E+c_{0 j} \pi_{j}}\left(\pi_{k}-c_{k j} \pi_{j}-c_{j k} \pi_{j}+c_{j k} c_{j l} \pi_{l}\right)-c_{0 k} .
$$

This same expression can also be found by calculating the group velocity $\vec{v}_{g}=\vec{\nabla}_{\vec{\pi}} E$. However, as previously stated, we will drop the $c_{0 j}$ contributions in much of the following and use

$$
v_{k}=\frac{1}{E}\left(\pi_{k}-c_{k j} \pi_{j}-c_{j k} \pi_{j}+c_{j k} c_{j l} \pi_{l}\right) .
$$

If rotation invariance is unbroken in the inertial frame we are considering, so that $c_{j k} \propto \delta_{j k}$, there is merely a rescaling of the velocity. This will lead to fewer interesting effects. Fortunately, even if there is a privileged frame in which $c_{j k} \propto \delta_{j k}$, a body emitting synchrotron radiation will not generally be at rest in this frame. We shall therefore assume that there is some breaking of rotation invariance in the rest frame of the source. In general, we shall assume that there is no suppression of rotation invariance violation relative to boost invariance violation only.

The equation of motion for the particle is the unmodified Lorentz force law $\dot{\vec{\pi}}=e \dot{\vec{x}} \times \vec{B}$. We shall neglect the Zitterbewegung in $\dot{\vec{x}}$ and consider only

$$
\dot{\vec{\pi}}=e \vec{v} \times \vec{B}
$$

Then, since according to (8) $\vec{v}$ remains a linear function of the momentum, we may solve for the particle's motion exactly. Again, we emphasize that all these same results for the bulk velocity and equation of motion also apply to Klein-Gordon particles, although the Klein-Gordon equation is even less satisfactory as a single-particle wave equation than is the Dirac equation.

It is advantageous to solve for the time development of the velocity, rather than the momentum (canonical or mechanical). While the velocity is a less fundamental object, the greatest formal problem with it - the Zitterbewegung - has already been neglected. The momentum could be determined with equal ease, but it possesses the unattractive property that a particle could possess zero momentum, yet not be stationary, if a $c_{0 j}$ term were present. Moreover, even with the Lorentz-violation, the electromagnetic field is coupled directly to the velocity. Because $c^{\nu 0}=0$, the electrostatic potential $\Phi=A^{0}$ is coupled, as usual, to the charge density $e \psi^{\dagger} \psi$. Similarly, the vector potential $\vec{A}$ couples 
to $e \psi^{\dagger} \dot{\vec{x}} \psi$, where $\dot{\vec{x}}$ is given by (4). Neglecting the Zitterbewegung, the coupling is simply to the bulk velocity $\vec{v}$. The fact that that the electromagnetic coupling is standard in this way was already evident in the Lorentz force law (9), and it holds equally in the equations of motion for $A^{\mu}$.

To determine the particle's motion, we must solve a set of two coupled differential equations. These two equations describe the time evolution of the two components of the velocity in the plane perpendicular to $\vec{B}$; the component of $\vec{v}$ parallel to $\vec{B}$ does not contribute to the $\vec{v} \times \vec{B}$ force. Let us take $\vec{B}$ to point along the $z$-direction, $\vec{B}=B \hat{z}$. Then the equations of motion for $\vec{\pi}$ are

$$
\begin{aligned}
& \dot{\pi}_{1}=e B v_{2} \\
& \dot{\pi}_{2}=-e B v_{1} \\
& \dot{\pi}_{3}=0 .
\end{aligned}
$$

So $\pi_{3}$ is a constant of the motion, as is $E=\sqrt{m^{2}+\left(\pi_{k}-c_{k j} \pi_{j}\right)\left(\pi_{k}-c_{k l} \pi_{l}\right)}$. Differentiating (8) then gives the following equations of motion for $v_{1}$ and $v_{2}$

$$
\begin{aligned}
& \dot{v}_{1}=\frac{1}{E}\left[\left(1-2 c_{11}+c_{j 1} c_{j 1}\right) \dot{\pi}_{1}+\left(-c_{12}-c_{21}+c_{j 1} c_{j 2}\right) \dot{\pi}_{2}\right] \\
& \dot{v}_{2}=\frac{1}{E}\left[\left(-c_{12}-c_{21}+c_{j 1} c_{j 2}\right) \dot{\pi}_{1}+\left(1-2 c_{22}+c_{j 2} c_{j 2}\right) \dot{\pi}_{2}\right] .
\end{aligned}
$$

Combining equations (10), (11), (13), and (14) in matrix form gives

$$
\left[\begin{array}{c}
\dot{v}_{1} \\
\dot{v}_{2}
\end{array}\right]=\frac{e B}{E}\left[\begin{array}{ll}
-\beta & \alpha \\
-\gamma & \beta
\end{array}\right]\left[\begin{array}{l}
v_{1} \\
v_{2}
\end{array}\right]=\omega_{0} M\left[\begin{array}{l}
v_{1} \\
v_{2}
\end{array}\right]
$$

The elements of the matrix $M$ are $\alpha=\left(1-2 c_{11}+c_{j 1} c_{j 1}\right), \beta=\left(-c_{12}-c_{21}+c_{j 1} c_{j 2}\right)$, and $\gamma=\left(1-2 c_{22}+c_{j 2} c_{j 2}\right)$, and $\omega_{0}=\frac{e B}{E}$.

The equation (15) is easily solved. Since $M^{2}=-\left(\alpha \gamma-\beta^{2}\right) I$ (where $I$ is the identity matrix), $e^{\omega_{0} M t}=I \cos \omega t+\frac{\omega_{0}}{\omega} M \sin \omega t$, where $\omega=\omega_{0} \sqrt{\alpha \gamma-\beta^{2}}$. For vanishing $c^{\nu \mu}, \omega=\omega_{0}$ is the usual synchrotron frequency. If we choose coordinates so that the initial conditions are $v_{1}(t=0)=v_{10}$ and $v_{2}(0)=0$, then

$$
\left[\begin{array}{l}
v_{1}(t) \\
v_{2}(t)
\end{array}\right]=e^{M t}\left[\begin{array}{c}
v_{10} \\
0
\end{array}\right]=v_{10}\left[\begin{array}{c}
\cos \omega t+\frac{\omega_{0}}{\omega}\left(c_{12}+c_{21}-c_{j 1} c_{j 2}\right) \sin \omega t \\
-\frac{\omega_{0}}{\omega}\left(1-2 c_{22}+c_{j 2} c_{j 2}\right) \sin \omega t
\end{array}\right] .
$$

The velocity in the $z$-direction can be found by direct integration of its derivative,

$$
\dot{v}_{3}=\frac{e B}{E}\left[\left(-c_{13}-c_{31}+c_{j 1} c_{j 3}\right) v_{2}+\left(c_{23}+c_{32}-c_{j 2} c_{j 3}\right) v_{1}\right] .
$$

So, if $v_{3}(0)=v_{30}$,

$$
\begin{aligned}
v_{3}(t)= & v_{10} \frac{\omega_{0}}{\omega}\left\{\left(c_{23}+c_{32}-c_{j 2} c_{j 3}\right)\left[\sin \omega t-\left(\frac{\omega_{0}}{\omega}\right)\left(c_{12}+c_{21}-c_{j 1} c_{j 2}\right)(\cos \omega t-1)\right]\right. \\
& \left.-\left(\frac{\omega_{0}}{\omega}\right)\left(c_{13}+c_{31}-c_{j 1} c_{j 3}\right)\left(1-2 c_{22}+c_{j 2} c_{j 2}\right)(\cos \omega t-1)\right\}+v_{30} .
\end{aligned}
$$


The particle moves in an elliptical helix; there is a constant drift parallel to $\vec{B}$, superimposed upon an additional periodic motion. If the drift vanishes, then the orbit lies close to, but is not generally in, the plane normal to the magnetic field, because $v_{3}$ does not generally vanish, even if its mean value does.

\section{Radiation Emission}

We shall now move on to the second stage our calculation. We have the particle's motion prescribed, so we may study the radiation emitted during this motion. For simplicity, we shall consider only the case in which the drift velocity is zero. [This does not correspond to $v_{30}=0$, because there are additional time-independent terms in (18). Instead, the sum of these constant terms must vanish.] However, since we have now formulated the problem in terms of a particle of prescribed velocity conventionally coupled to the radiation field, normal boosting techniques can be used to generalize these results to a situation in which the time-averaged velocity in the $z$-direction is nonvanishing. A crucial quantity to calculate is the speed of the particle, $|\vec{v}|$, which is given by

$$
\begin{aligned}
\vec{v}^{2} & =\frac{v_{10}^{2}}{2}[(\eta+\xi)+(\eta-\xi) \cos 2 \omega t+\zeta \sin 2 \omega t] \\
& =\frac{v_{10}^{2}}{2}\left[(\eta+\xi)+\sqrt{(\eta-\xi)^{2}+\zeta^{2}} \cos (2 \omega t-2 \phi)\right],
\end{aligned}
$$

where $\tan 2 \phi=\zeta /(\xi-\eta)$ and the constants $\eta, \xi$, and $\zeta$ are

$$
\begin{aligned}
\eta= & 1+\left(\frac{\omega_{0}}{\omega}\right)^{4}\left[\left(c_{23}+c_{32}-c_{j 2} c_{j 3}\right)\left(c_{12}+c_{21}-c_{j 1} c_{j 2}\right)\right. \\
& \left.+\left(c_{13}+c_{31}-c_{j 1} c_{j 3}\right)\left(1-2 c_{22}+c_{j 2} c_{j 2}\right)\right]^{2} \\
\xi= & \left(\frac{\omega_{0}}{\omega}\right)^{2}\left[\left(c_{12}+c_{21}-c_{j 1} c_{j 2}\right)^{2}+\left(1-2 c_{22}+c_{j 2} c_{j 2}\right)^{2}+\left(c_{23}+c_{32}-c_{j 2} c_{j 3}\right)^{2}\right] \\
\zeta= & 2 \frac{\omega_{0}}{\omega}\left(c_{12}+c_{21}-c_{j 1} c_{j 2}\right)-2\left(\frac{\omega_{0}}{\omega}\right)^{3}\left(c_{23}+c_{32}-c_{j 2} c_{j 3}\right) \\
& \times\left[\left(c_{23}+c_{32}-c_{j 2} c_{j 3}\right)\left(c_{12}+c_{21}-c_{j 1} c_{j 2}\right)+\left(c_{13}+c_{31}-c_{j 1} c_{j 3}\right)\left(1-2 c_{22}+c_{j 2} c_{j 2}\right)\right]
\end{aligned}
$$

Thus far, our results have been exact, except that we have neglected the radiative reaction force. Henceforth, we shall be making use of the standard, Lorentz-invariant results on the power radiated by a particle undergoing synchrotron motion [40. However, the standard methodology for evaluating synchrotron emission involves a number of approximations. One often neglects any effect suppressed by a positive power of the Lorentz factor $\gamma$, and we shall follow this prescription. Among the things we may therefore neglect is the radiation due to the component of the acceleration parallel to the velocity; this contribution to the emission is small in comparison with that arising from the perpendicular 
component of the acceleration. We may also ignore the angular width of the radiation beam. All the emitted energy is beamed into a narrow pencil of angles centered around the instantaneous direction of the velocity. The range of angles covered is $\mathcal{O}\left(\gamma^{-1}\right)$, but we shall neglect this spread, instead assuming that all radiation is emitted along a ray tangent to the particle's path.

We shall also neglect the Lorentz violation as a source of angular deviation. Although the exact orbit is neither circular nor in the plane normal to $\vec{B}$, the deviations from the conventional trajectory are small, of $\mathcal{O}(c)$. It would not be feasible to measure changes in the angular distribution of the emitted radiation induced by the presence of the Lorentz violation. We shall therefore neglect the changes in the orbital shape. All effects we shall consider will therefore be related to the modification of $|\vec{v}|(20)$. (This is similar to the approach adopted in [29], where the magnitude of the velocity was also taken as the central quantity.) As the velocity changes around the particle's nearly circular path, the rate at which radiation is emitted will vary. The most sensitive tests of $c$-type Lorentz violation in synchrotron radiation could come from comparing the power output in different directions. (Unfortunately, such measurements are obviously not possible for single astrophysical sources.)

The phase $\phi$ represents the angular position of the particle in its orbit at the time when the velocity is a maximum. At the antipodal point of the orbit, the velocity is also maximal. The greatest amount of radiation is then emitted along the tangent rays at these two points and propagates in the directions given by the azimuthal angles $\phi \pm \frac{\pi}{2}$. Similarly, the smallest radiated power is in the directions $\phi$ and $\phi+\pi$. The presence of this effect is of course dependent on the existence of rotation invariance violation.

Neglecting radiation due to the component of the acceleration parallel to the velocity [which is smaller by a factor of $\mathcal{O}\left(\gamma^{-2}\right)$ ], the intensity spectrum per unit spectral frequency $\omega_{s}$ is

$$
\frac{d I}{d \omega_{s}}=\sqrt{3} e^{2} \gamma \frac{\omega_{s}}{\omega_{c}} \int_{\omega_{s} / \omega_{c}}^{\infty} d x K_{5 / 3}(x) .
$$

The critical frequency is $\omega_{c}=\frac{3}{2} \gamma^{3} \rho^{-1}$, and $\rho$ is the instantaneous radius of curvature of the orbit $\rho=\vec{v}^{2} /\left|\vec{a}^{\perp}\right|$, where $\vec{a}^{\perp}$ is the component of the acceleration perpendicular to $\vec{v}, \vec{a}^{\perp}=\dot{\vec{v}}-\frac{\dot{\vec{v}} \cdot \vec{v}}{\vec{v}^{2}} \vec{v}$. Neglecting the Lorentz-violating corrections, $\rho$ is approximately $E /|e| B . K_{5 / 3}(x)$ is a modified Bessel function of the second kind. One could go further and calculate the radiation fields in the far field explicitly. However, we shall not do this, because the polarization structure of the emitted radiation is not substantially effected by the Lorentz violation.

For ultrarelativistic particles, for which $1-|\vec{v}| \ll 1$, the Lorentz factor is roughly $\gamma \approx 1 / \sqrt{2(1-|\vec{v}|)}$, and this is a rapidly increasing function of the speed- $d \gamma / d|\vec{v}|=$ $|\vec{v}| \gamma^{3} \approx \gamma^{3}$. The description of the Lorentz violation through an effective field theory containing only $c^{\nu \mu}$ terms will break down if the modifications of the velocity due to the presence of $c$ can render the speed superluminal. According to (8), this can occur when 
$|\vec{\pi}| / E \approx 1-|c|$, where $|c|$ is a characteristic size for the Lorentz-violating coefficients. This gives us an estimate of the maximum value of $\gamma$ that can be achieved before new physics must come into play if some form of causality is to be preserved: $\gamma_{\max } \sim 1 / \sqrt{|c|}$. This corresponds to an energy scale $E_{\max } \sim \sqrt{m M_{P}}$.

\section{Prospects for Observability}

It is still unclear whether the changes we have described in the emission will be observable, and we shall now turn our attention to this issue. The total radiated synchrotron power in the ultrarelativistic regime is proportional to $\gamma^{4}$. Therefore, the change in the radiated power as the velocity varies around the orbit is given by

$$
\frac{\Delta P}{P} \approx 2 \frac{\left[d\left(\gamma^{4}\right) / d|\vec{v}|\right]|c|}{\gamma^{4}} \approx 8 \gamma^{2}|c| .
$$

The factor of 2 comes from the fact that the deviations in $|\vec{v}|$ range over both positive and negative values. The characteristic size $|c|$ used in this calculation should be essentially the same as that used in the determination of $\gamma_{\max }$, because in both cases, $|c|$ measures the magnitude of the contribution that $c^{\nu \mu}$ can make to the velocity. Inserting $\gamma=\gamma_{\max }$ into (25) gives a result that is greater than unity. This means that the fractional change in the emitted power can be of order one in the regime in which the theory is valid; we do not have to go to an energy scale so high that new physics must emerge in order to see changes in the emission. However, we do need to get comparatively close to the scale at which the theory breaks down in order to observe deviations in the spectrum.

In fact, for $|c| \approx 10^{-19}$, we will find a $\frac{\Delta P}{P}$ of one percent at $\gamma \approx 10^{8}$. For the lightest charged particle, the electron, this corresponds to an energy of roughly $50 \mathrm{TeV}$, orders of magnitude beyond anything one could create in the laboratory. We conclude that these effects are unobservable for Earth-based sources.

The only sources of synchrotron radiation that are high enough in energy to give observable results of the type we are considering are astrophysical. However, as each astrophysical source can only be observed from a single direction, more than one source would be required in order to make the kind of directional observations that could constrain $c$ most strongly. Ideally, we would want to have two or more very clean sources of synchrotron radiation, for which the spectra due to the motion of multiple species of particles (e.g., both electrons and protons) could be resolved. Then we could look for systematic differences between the emission profiles for the species. This would mean effectively using the proton spectra, for example, as local magnetometers and looking to see whether the electron spectra are consistent with the measured fields. One could then set bounds on a combination of the $c$ coefficients for the electron and the proton.

Although observations of distant synchrotron sources (such as far-off radio galaxies) are ideal for constraining Lorentz violation in the photon sector, they are not so helpful 
here. A long line of sight will magnify small effects that modify the propagation structure of radiation. However, large distances do nothing to assist measurements of Lorentz violation in the charged emitters themselves. A nearby, accurately understood source is better than a distant one.

The best-understood synchrotron source is the Crab nebula, but its spectrum still appears too complicated for the kind of procedure we have suggested to be at all feasible. (For a good review of the Crab nebula's nonthermal emission spectrum, see [43.) For example, the spectrum contains two different electron synchrotron components, with significantly different characteristics. Any observed proton synchrotron radiation would probably fail as a sensitive magnetometer, because it would be impossible to associate it uniquely and in a model-independent fashion with either one or the other electron population. Based on measurements of the entire spectrum, the average strength of the magnetic field in the x-ray production region is known to be in the tenths of $\mathrm{mG}$, but it is not known to high accuracy. There are also large relative uncertainties in the radiation rates in some regimes, particularly the highest energy.

However, we still can get a strong constraint on $c$ from the Crab nebula data. This constraint, like the one derived in [29], is based upon the fact that Lorentz violation may give rise to a maximum particle velocity. The existence of electrons with large velocities then constrains the Lorentz-violating parameters. For a particle moving in the direction of a unit vector $\vec{e}$, the maximum allowed velocity is (to leading order in c) $1-c_{j k} e_{j} e_{k}-c_{0 j} e_{j}$. We observe via the Crab nebula synchrotron spectrum electrons with Lorentz factors as large as $3 \times 10^{9}$. This means that the maximum velocity in the Crab-to-Earth direction is greater than $1-6 \times 10^{-20}$, hence $c_{j k} e_{j} e_{k}+c_{0 j} e_{j}<6 \times 10^{-20}$. As in [29], this is a one-sided limit; one sign of this combination of coefficients leads to a maximum velocity in the relevant direction, but the other does not.

The direction $\vec{e}$ can be transformed into the standard sun-centered celestial equatorial coordinates used in the study of Lorentz violation [41. The location of the Crab nebula is right ascension $5 \mathrm{~h} 34 \mathrm{~m} 32 \mathrm{~s}$, declination $22^{\circ} 0^{\prime} 52^{\prime \prime}$, lying close to the ecliptic plane. So the unit vector pointing from the nebula to the Earth has components $e_{X}=-0.10$, $e_{Y}=-0.92$, and $e_{Z}=-0.37$. This gives us the particular elements of the $c$ tensor that are constrained by this measurement. The specific constraint is

$$
\begin{aligned}
{\left[0.01 c_{X X}+\right.} & 0.85 c_{Y Y}+0.14 c_{Z Z}+0.09 c_{(X Y)}+0.04 c_{(X Z)} \\
& \left.+0.34 c_{(Y Z)}-0.10 c_{0 X}-0.92 c_{0 Y}-0.37 c_{0 Z}\right]<6 \times 10^{-20},
\end{aligned}
$$

where $c_{(j k)}$ is the symmetric sum $c_{j k}+c_{k j}$. Similar constraints could be obtained for other well-resolved synchrotron sources; this would provide further constraints on the symmetric part of $c_{j k}$ and on the $c_{0 j}$. [At leading order, the antisymmetric part of $c_{j k}$ just represents a change in the representation of the Dirac matrices, and it is already evident from (77) that it will not contribute to the velocity.]

In order for these constraints to be valid, we must know that there are no other effects 
that will interfere with our result. In particular, we would like to address the question of whether quantum corrections would affect the emission before Lorentz-violating corrections become important. The leading order quantum corrections to the standard synchrotron formulas may be found by making the replacement $\omega_{s} \rightarrow \omega_{s}\left(1+\frac{\omega_{c}}{E}\right)$ in $\frac{1}{\omega_{s}} \frac{d I}{d \omega_{s}}$ [42]. The corrections are negligible if $\omega_{c} \ll E$, or equivalently if $\gamma \ll \frac{m^{2}}{|e| B}$. This is $\gamma \ll\left(3 \times 10^{13}\right) B^{-1}$ if the particle is an electron and $B$ is measured in Gauss. If a typical field strength is that within the Crab nebula, $B \sim 0.2-0.3 \mathrm{mG}$, the maximum values of $\gamma$ are extremely high. So our classical treatment could apply up to scales well above those at which we would expect to start seeing marked deviations from the conventional results.

Synchrotron radiation has already been used to set strong limits on nonrenormalizable Lorentz-violating modifications of quantum electrodynamics. Lorentz violation in synchrotron radiation is also theoretically interesting, and there have been a number of prior analyses of the emission spectrum in specific Lorentz-violating models. In this paper, we have looked at the impact of the renormalizable SME coefficient $c^{\nu \mu}$ on synchrotron processes. Although we have used a number of standard approximations to simply our analysis of the radiation, no approximations relating to the Lorentz violation were required; the expressions (16) and (18) are exact, valid to all orders in $c$. The $c^{\nu \mu}$ coefficients for electrons, particular the diagonal coefficients, can be difficult to bound experimentally [17, 41]. Since only a single fermion is involved in synchrotron radiation, this is a process in which it is relatively easy to isolate electron-specific effects, and the kind of constraints we have obtained here should prove useful.

\section{Acknowledgments}

The author is grateful to V. A. Kostelecký for helpful discussions. This work is supported in part by funds provided by the U. S. Department of Energy (D.O.E.) under cooperative research agreement DE-FG02-91ER40661.

\section{References}

[1] D. Colladay, V. A. Kostelecký, Phys. Rev. D 55, 6760 (1997).

[2] D. Colladay, V. A. Kostelecký, Phys. Rev. D 58, 116002 (1998).

[3] V. A. Kostelecký, Phys. Rev. D, 69105009 (2004).

[4] V. A. Kostelecký, C. D. Lane, A. G. M. Pickering, Phys. Rev. D 65, 056006 (2002).

[5] V. A. Kostelecký, R. Lehnert, Phys. Rev. D 63, 065008 (2001).

[6] R. Bluhm, V. A. Kostelecký, N. Russell, Phys. Rev. Lett. 79, 1432 (1997). 
[7] R. Bluhm, V. A. Kostelecký, N. Russell, Phys. Rev. D 57, 3932 (1998).

[8] G. Gabrielse, A. Khabbaz, D. S. Hall, C. Heimann, H. Kalinowsky, W. Jhe, Phys. Rev. Lett. 82, 3198 (1999).

[9] H. Dehmelt, R. Mittleman, R. S. Van Dyck, Jr., P. Schwinberg, Phys. Rev. Lett. 83, 4694 (1999).

[10] R. Bluhm, V. A. Kostelecký, N. Russell, Phys. Rev. Lett. 82, 2254 (1999).

[11] D. F. Phillips, M. A. Humphrey, E. M. Mattison, R. E. Stoner, R. F. C. Vessot, R. L. Walsworth, Phys. Rev. D 63, 111101 (R) (2001).

[12] R. Bluhm, V. A. Kostelecký, C. D. Lane, Phys. Rev. Lett. 84, 1098 (2000).

[13] V. W. Hughes, et al., Phys. Rev. Lett. 87, 111804 (2001).

[14] R. Bluhm, V. A. Kostelecký, Phys. Rev. Lett. 84, 1381 (2000).

[15] B. Heckel, et al., in Elementary Particles and Gravitation, edited by B. N. Kursonoglu, et al. (Plenum, New York, 1999).

[16] C. J. Berglund, L. R. Hunter, D. Krause, Jr., E. O. Prigge, M. S. Ronfeldt, S. K. Lamoreaux, Phys. Rev. Lett. 75, 1879 (1995).

[17] V. A. Kostelecký, C. D. Lane, Phys. Rev. D 60, 116010 (1999).

[18] D. Bear, R. E. Stoner, R. L. Walsworth, V. A. Kostelecký, C. D. Lane, Phys. Rev. Lett. 85, 5038 (2000).

[19] V. A. Kostelecký, Phys. Rev. Lett. 80, 1818 (1998).

[20] V. A. Kostelecký, Phys. Rev. D 61, 016002 (2000).

[21] Y. B. Hsiung, Nucl. Phys. Proc. Suppl. 86, 312 (2000).

[22] K. Abe et al., Phys. Rev. Lett. 86, 3228 (2001).

[23] S. M. Carroll, G. B. Field, R. Jackiw, Phys. Rev. D 41, 1231 (1990).

[24] S. M. Carroll, G. B. Field, Phys. Rev. Lett. 79, 2394 (1997).

[25] V. A. Kostelecký, M. Mewes, Phys. Rev. Lett. 87, 251304 (2001).

[26] R. C. Myers, M. Pospelov, Phys. Rev. Lett. 90, 211601 (2003).

[27] R. Montemayor, L. F. Urrutia, Phys. Lett. B 606, 86 (2005). 
[28] R. Montemayor, L. F. Urrutia, hep-ph/0505135.

[29] T. Jacobson, S. Liberati, D. Mattingly, Nature 424, 1019 (2003).

[30] T. Jacobson, S. Liberati, D. Mattingly, F. W. Stecker, Phys. Rev. Lett. 93, 021101 (2004).

[31] J. R. Ellis, N. E. Mavromatos, A. S. Sakharov, Astropart. Phys. 20, 669 (2004).

[32] S. M. Carroll, J. A. Harvey, V. A. Kostelecký, C. D. Lane, T. Okamoto, Phys. Rev. Lett. 87, 141601 (2001).

[33] P. Castorina, A. Iorio, D. Zappalà, Phys. Rev. D 69, 065008 (2004).

[34] R. Lehnert, R. Potting, Phys. Rev. Lett. 93, 110402 (2004).

[35] R. Lehnert, R. Potting, Phys. Rev. D 70, 125010 (2004).

[36] V. A. Kostelecký, M. Mewes, Phys. Rev. D 66, 056005 (2002).

[37] Q. G. Bailey, V. A. Kostelecký, Phys. Rev. D 70, 076006 (2004).

[38] R. Lehnert, J. Math. Phys. 45, 3399 (2004).

[39] B. Altschul, D. Colladay, Phys. Rev. D 71, 125015 (2005).

[40] J. Schwinger, Phys. Rev. 75, 1912 (1949).

[41] R. Bluhm, V. A. Kostelecký, C. D. Lane, N. Russell, Phys. Rev. D 68, 125008 (2003).

[42] J. Schwinger, Proc. Natl. Acad. Sci. 40, 132 (1954).

[43] F. A. Aharonian, A. M. Atoyan, 1998, in Neutron Stars and Pulsars, edited by N. Shibazaki, N. Kawai, S. Shibata, T. Kifune (Universal Academy Press, Tokyo, 1998), p. 439 astro-ph/9803091. 